\title{
Anatomía de los órganos vegetativos y reproductivos de Crescentia alata y Crescentia cujete (Bignoniaceae)
}

\author{
Tania Paola López, Lucy López Arteaga' \\ Lilian Ferrufino ${ }^{2}$
}

\section{RESUMEN}

Crescentia es un género que habita en los bosques secos y en Centroamérica estas especies son consideradas de mucha importancia económica. La separación taxonómica de $C$. alata y $C$. cujete ha sido muy discutida, ya que ambas especies son cercanamente relacionadas.

El objetivo de este estudio es conocer la anatomía vegetativa y reproductiva de $C$. alata y $C$. cujete. Entre las características anatómicas observadas en ambas especies están la presencia de peridermis y una médula con fibras de floema secundario en los tallos; la hoja presenta nectarios extraflorales y en la vena media un mesófilo dorsiventral con parénquima en empalizada y lagunoso; también, en el cilindro vascular se observan fibras perifloemáticas; los pétalos y sépalos muestran muchas acumulaciones de pigmentos y abundantes pelos glandulares, nectarios florales y el polen tiene una ornamentación colpado; la semilla presenta una gran cantidad de endospermo y una capa de aleurona.

En conclusión, ambas especies tienen características anatómicas similares, sin embargo, la ornamentación de los granos polen es diferente entre ambas especies, en particular el número de colpas.

Palabras clave: Crescentia, anatomía, nectarios, extraflorales, florales, polen.

\section{ABSTRACT}

Crescentia is a genus that inhabits in dry forests, and Central America the species are considered to have great economic importance. The taxonomic separation of $C$. alata and $C$. cujete has been much debated, since both species are closely related. The aim of this study was to determine the vegetative and reproductive anatomy of C. alata and C. cujete. Among the anatomical characteristics observed in both

\footnotetext{
${ }^{1}$ Estudiantes de la Carrera de Biología, Departamento de Biología, Facultad de Ciencias, UNAH.

${ }^{2}$ Asesora, coordinadora de investigación y docente del Departamento de Biología, Facultad de Ciencias, UNAH; dirección electrónica: ferrufinolilian@yahoo.com
} 
species, are: the presence of periderm and pith with secondary phloem fibers in stems; the leaf has extrafloral nectaries, and in the midrib possess dorsiventral mesophyll with palisade parenchyma and lacune tissue also the vascular cylinder has periphloematics fibers; petals and sepals possess many accumulations of pigment and abundant glandular hairs, floral nectaries and pollen has colpate ornamentation; and the seed has a large amount of endosperm and aleurone layer. In conclusion, both species have similar anatomical characteristics; however, the pollen grains ornamentation is different between the two species in particular, the number of colpus or apertures.

Key Words: Crescentia, anatomy, nectaries, extrafloral, floral, pollen.

\section{INTRODUCCIÓN}

Los árboles del género Crescentia de la familia Bignoniaceae, presentan una abundancia muy amplia en el bosque seco tropical caracterizado por la predominancia de matorrales y arbustos con espinas, las cuales utilizan como mecanismo de defensa ante las largas estaciones secas. En toda América se les conoce con diferentes nombres vernáculos, pero en Honduras se le llama jícaro 0 jícara; su vida oscila entre los 100 y 200 años, presentan una forma irregular, copa ligera y muchos intersticios entre las ramas, estas siguen un patrón dicotómico en su tronco y a medida que crecen se dividen a su vez en dos (Jiménez, 2008; Jarquín, 2012).

Crescentia alata Kunth es un árbol que crece de manera silvestre en el bosque seco de Honduras. Mide más de 10 metros de altura, con numerosas ramas de 6-10 metros de largo, las hojas son trifoliadas con pecíolo alado. Se propaga por semillas o estacas, las flores nacen directamente del tronco, los frutos son redondeados de cáscara dura y pulpa blanda rodeada de muchas semillas de forma acorazonada, los cuales se colectan de manera silvestre cuando están maduros (ver figura 1). Los árboles de jícaro fructifican durante todo el año, pero su mayor producción es entre marzo y mayo (Hernández y Campos, 2007).

Crescentia cujete L., llamado también "morro guacalero", alcanza una altura de aproximadamente de $10 \mathrm{~m}$ y el tronco posee un diámetro de $20 \mathrm{~cm}$ con largas ramas extendidas. Las hojas son oblanceoladas o facioladas de $6-15 \mathrm{~cm}$ de largo, agudas, obtusas o cortantes acuminadas en el ápice, estrechamente hacia la base casi sésil. Las flores son grandes, solitarias y agrupadas, laterales o axilares; el pedúnculo es robusto con un cáliz coriáceo cerrado en el botón, 2-partido, 05hendido en la anterior; de 2-2.5 cm de largo, sus lóbulos anchos redondeados u 
obtusos. Presenta una corola de color purpura-amarillento de 5-6 cm de largo, sus lóbulos lanceolados muchos más cortos que el tubo subcampanulado; posee 4 estambres, didinamos, incluso un poco saliente y un disco anular. Posee un ovario 1locular, sésil, numerosos lóculos sobre dos placentas parietales. El fruto es globoso hasta elipsoide con una cáscara dura indehiscente (ver figura 1), con semillas numerosas, no aladas, comprimidas, insertas en placenta esponjosa (Simaj y García, 2004).

Las flores de Crescentia son polinizadas por murciélagos e insectos nocturnos y se presenta parasitismo floral por abejas (Partamona bilineata y Trigona fulviventris). Estas remueven aproximadamente el $40 \%$ de la producción total de néctar antes de la llegada del polinizador. El robo de polen afecta la fecundidad de la planta. La competencia por la atracción de polinizadores ha sido una importante fuerza evolutiva que ha modelado los sistemas sexuales de las plantas (Jarquín, 2012).

Crescentia alata y C. cujete son consideradas especies de mucha importancia económica. En Centroamérica se registran el uso culinario de la semilla que se extrae del fruto para la preparación de una bebida llamada horchata o morro (Chízmar, 2009). En Honduras, la cáscara de la fruta se usa para aliviar la tos, el asma y el dolor de estómago; las semillas son usadas para la disentería (House, 1995).

No se reporta ningún trabajo que trate de manera inclusiva los caracteres anatómicos del género Crescentia, sin embargo, sí existen estudios anatómicos a nivel de subfamilia y género que ayudan a la disociación de especies. Zapater y otros (2009) revisaron las especies argentinas de Tabebuia y Handroanthus y utilizaron el indumento como uno de los caracteres de importancia taxonómica. Similar estudio fue realizado por Seibert (1948) con respecto a las estructuras glandulares, enfatizando la importancia de este carácter y proponiendo una clasificación de las estructuras secretoras en la familia Bignoniaceae. Rivera (2000) señala la existencia de glándulas en la base del ovario. También se han realizados estudios de palinología como herramienta para identificar géneros en las subtribus de la familia (Gentry y Tomb, 1979).

El presente estudio tiene como objetivo describir la anatomía del tallo, hoja, flor y semilla de Crescentia alata y Crescentia cujete con el fin de identificar dos especies cercanamente relacionadas. 


\section{METODOLOGÍA}

Se colectó fruto, tallo, hojas y flores de las especies $C$. alata y C. cujete en Ajuterique, Comayagua, y en la Colonia Kennedy en Tegucigalpa. Se realizaron cortes transversales con hojas de afeitar a mano alzada en tallo, hojas, pétalos, fruto y semillas; se observaron granos de polen.

Se preservaron los cortes en glicerina para evitar la deshidratación temporal, luego se observaron en el microscopio compuesto los cortes realizados, identificando las estructuras presentes. Se hicieron cuadros comparativos entre las estructuras vegetativas y reproductivas de las especies y se relacionaron con las características ecológicas.

\section{RESULTADOS}

\section{Anatomía del tallo}

En el corte transversal del tallo se observa peridermis con una delgada capa de súber y un ritidoma anular, tejido muerto que está fuera del súber. En la médula se observan fibras de floema secundario (FFS), llamado líber o floema duro; además, floema, xilema secundario y cambium formado por meristemo lateral. Se observan radios medulares de 102 series de células (ver figuras 2 e y $3 e$ ).

\section{Anatomía de la hoja}

La hoja es trilobulada u ovalada. Se observa la epidermis con varias capas de células y en la superior tricomas glandulares. En la vena media de la hoja observa un mesófilo dorsiventral con parénquima en empalizada y lagunoso; además, el cilindro vascular presenta fibras perifloemáticas, es decir, células del esclerénquima que rodea al floema. También se observan células de xilema que tiene como función conducir agua y sales y colénquima que funciona como tejido de sostén.

Entre las características que se destacan está la presencia de los nectarios extraflorales o extranupciales en ambas especies. Los nectarios son discoides y su función es secretar néctar (ver figuras 2 d y 3 d).

\section{Anatomía de la flor}

Los pétalos y sépalos presentan muchas acumulaciones de pigmentos y 
abundantes pelos glandulares que secretan aceites y olores. En la epidermis superior de los sépalos y pétalos se observan nectarios florales, llamados nectarios nupciales (ver figuras 2 a, 2 b, 3 a y 3 b). El polen tiene una ornamentación colpado y porado. En C. alata, el polen presenta dos o tres colpas y en $C$. cujete posee solo una colpa o ecolpado (ver figura $2 \mathrm{c}$ ).

\section{Anatomía de la semilla}

El fruto inmaduro es una baya de color verde, que se torna de color verde amarillento a café cuando alcanza la madurez, su tamaño es variable. La semilla presenta una gran cantidad de endospermo (ver figuras $2 \mathrm{fy} 3 \mathrm{f}$ ) en ambas especies. La testa de la semilla presenta una capa de aleurona.

\section{DISCUSIÓN}

Anatomía de tallo

La característica más notable en la corteza de las especies de Bignoniaceae es el líber duro compuesto exclusivamente de fibras, así como radios medulares formados de una o dos series de células (Roth, 1969). Ambas características coinciden con las estructuras observadas en las dos especies de Crescentia.

Anatomía de hoja

Las características más destacable en $C$. alata y $C$. cujete es la presencia de una epidermis múltiple llamada hipodermis, la que proporciona resistencia mecánica en particular en plantas xeromórficas, es decir, que habitan en lugares secos y que tienen como función disminuir la intensidad de la luz que alcanza el tejido fotosintético. Esta característica ha sido encontrada en otras especies de los géneros de la familia Bignonicaeae, tales como: Handroanthus, Tabebuia, Catalpa, Pandorea, Tecoma, Campsis y Podranea (González, 2013).

Los nectarios extraflorales han sido observados en otras especies de la familia Bignoniaceae; González (2013) observó una secreción clara en plantas frescas y estas eran visitadas por hormigas en busca de néctar.

Anatomía de flor

Los nectarios florales observados en los pétalos son usados como carácter para identificar especies. Seibert (1948) y Rivera (2000) reportan nectarios nupciales o 
florales en otras especies de la familia Bignoniaceae y proponen que la forma de estas estructuras es útil para identificar plantas de esta familia a nivel género.

La exina del polen de ambas especies es similar, varios autores señalan que Crescentia alata y $C$. cujete que se distribuyen en Centroamérica se consideran especies difíciles de separar palinológicamente. Gentry y Tomb (1979) comentan que las dos especies aparentemente se hibridan en la naturaleza y el desafío de validar su segregación específica podría ser un fundamento muy fuerte más que su separación taxonómica a nivel de género.

Varios autores han descrito la ornamentación de C. alata. Urban (1916) la describe sin apertura y Mitra (1968) lo detalla multiporado. Sin embargo, nuestros resultados concuerdan con Gentry (1979), quien describe el polen de C. alata como tricolpado y C. cujete con una colpa y o sin colpa.

\section{Anatomía de la semilla}

Las semillas de C. alata y $C$. cujete son de crecimiento ortodoxo, pequeñas y aplanadas; la testa es de color café oscuro a negro, presenta dos cotiledones y sin endospermo. Según Solares (2004), Crecentia alata se considera un recurso usado para forrajero y alimento, dado que la pulpa y la semilla contienen altos valores de proteínas y carbohidratos y sin la presencia de toxinas como taninos, saponinas y alcaloides. También Meyrat (1983), menciona que en las semillas se concentra la mayor cantidad de proteína con un $24.12 \%$, aceites $21 \%$ y carbohidratos $43 \%$; en la pulpa se concentra la mayor cantidad de agua con un $79.17 \%$ y de 10 a $15 \%$ de carbohidratos.

\section{CONCLUSIONES}

La anatomía vegetativa y reproductiva de Crescentia alata y Crescentia cujete es similar, no obstante, existe diferencias en la ornamentación del polen, ya que $C$. alata presenta un polen tricolpado, mientras que $C$. cujete tiene una colpa.

Con base en los comentarios de Gentry y Tomb (1979), es posible afirmar que estas dos especies supuestamente se hibridan en la naturaleza y el desafío de validar su segregación disociación a nivel de especie podría ser un fundamento muy fuerte más que su separación taxonómica a nivel de género. Sin embargo, se recomienda hacer un estudio más detallado sobre la biología reproductiva de ambas especies. 
Figura 1. Especies de Crescentia

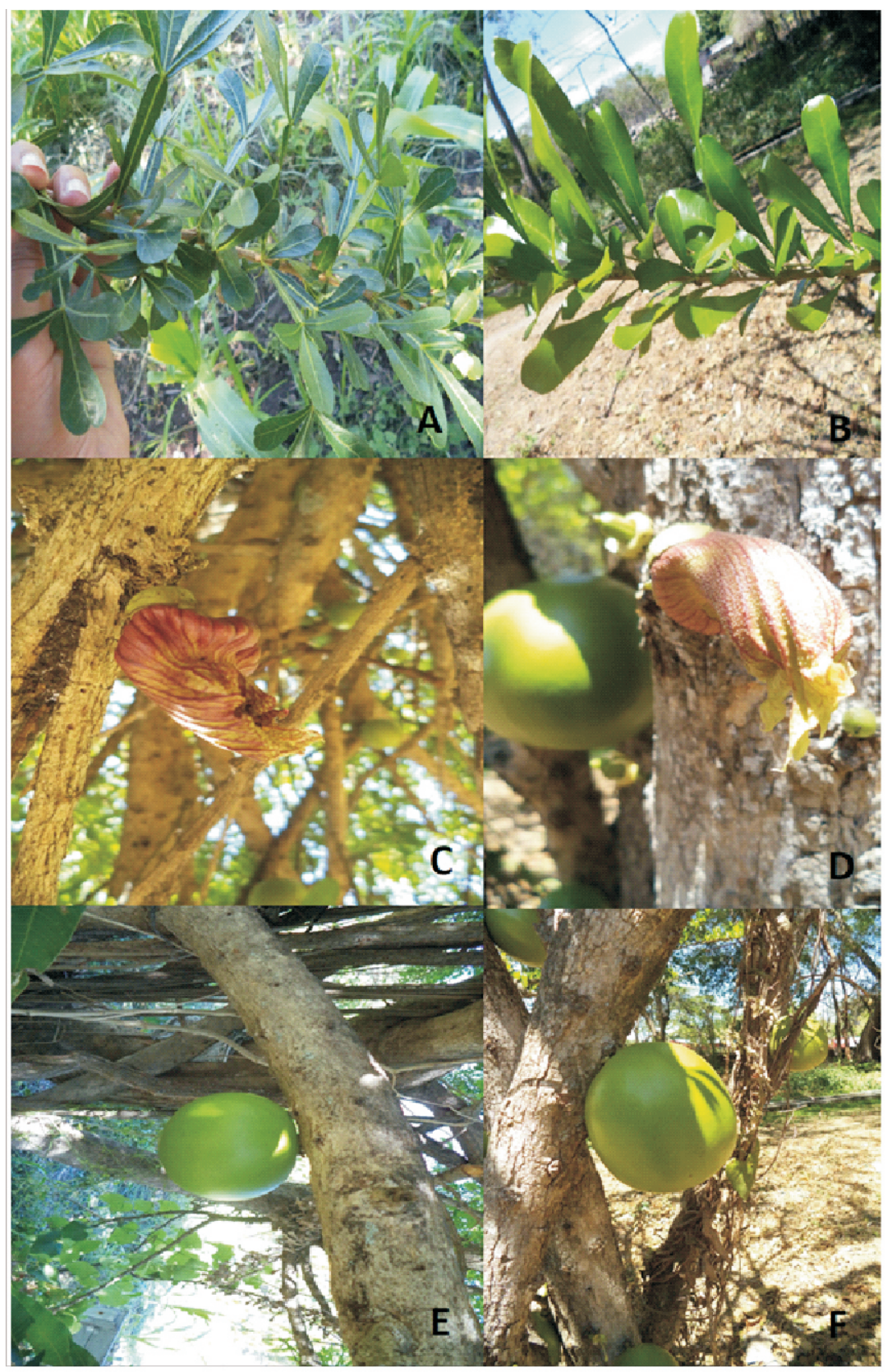

$A, C$ y E: Crescentia alata; $B, D$ y F: Crescentia cujete. 
Figura 2. Anatomía de órganos vegetativos y reproductivos de Crescentia alata

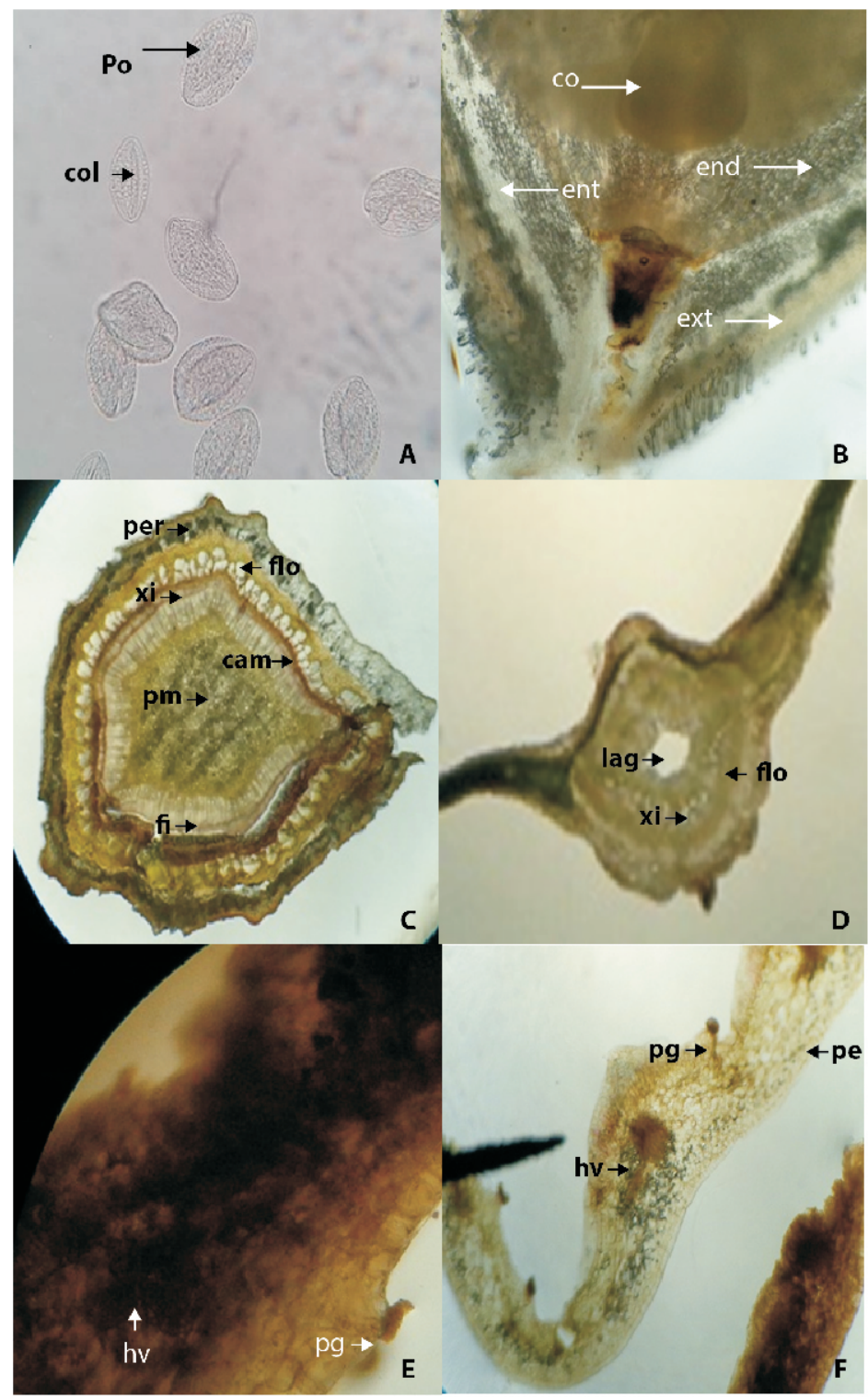

A: Granos de polen con ornamentación colpada y porado. B: Corte longitudinal de semilla con cubierta externa dura. C: Corte transversal de tallo en el que se observa la gran cantidad de rayos. D: Corte transversal de hoja. E: Corte transversal de sépalo. F: Corte transversal de pétalo. Abreviaturas: po, poros; col, colpas; co, cotiledones; end, endospermo; ent, endotesta; ext, exotesta; per, peridermis; cam, cambium; xi, xilema; flo, floema; fi, fibra; pm, parénquima medular; lag, laguna; hv, haz vascular; pg, pelo glandular; pe, parénquima esponjoso. 
Figura 3. Anatomía de órganos vegetativos y reproductivos de Crescentia cujete

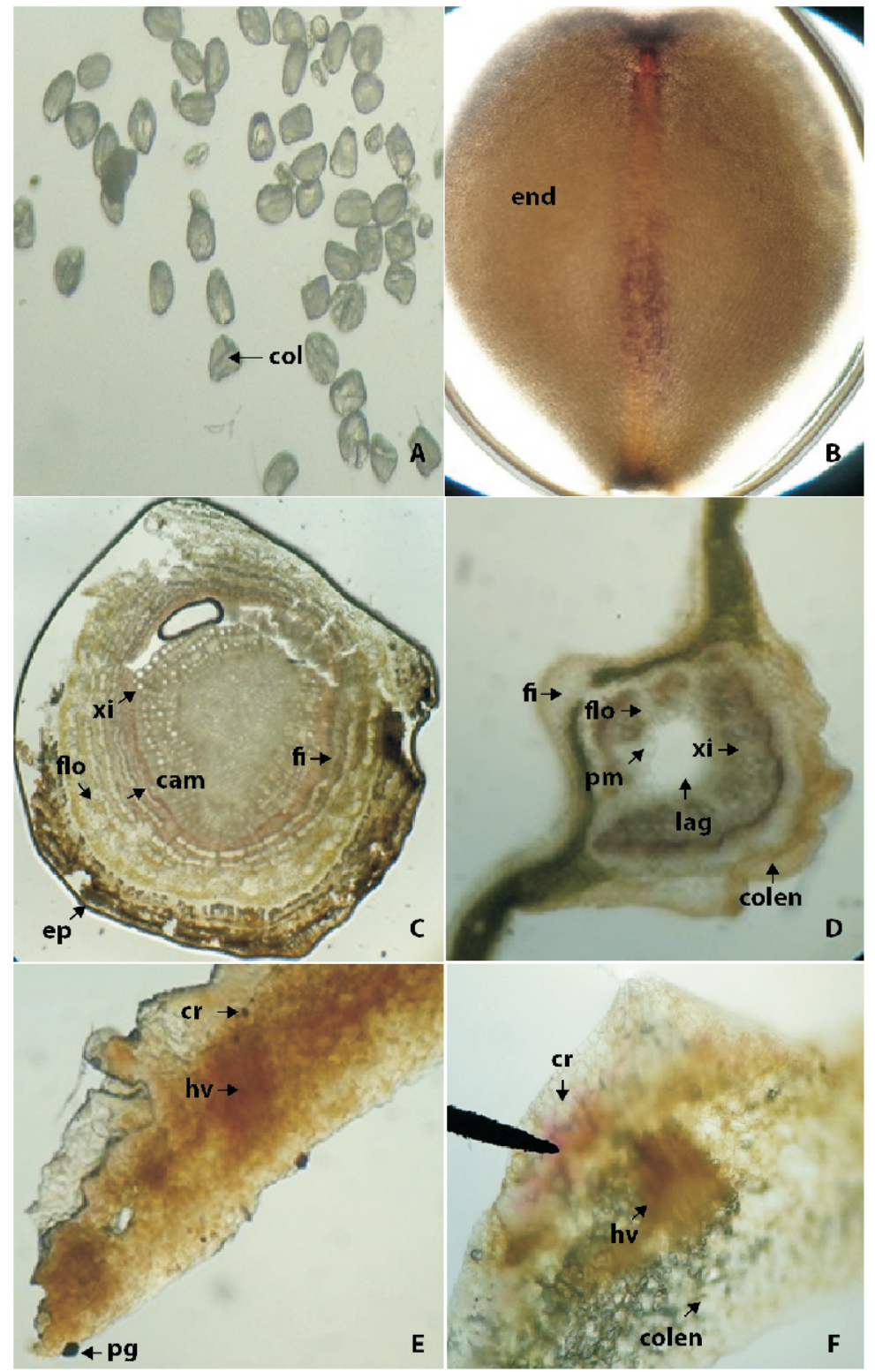

A: Granos de polen polimórficos y con una colpa. B: Corte longitudinal de semilla de textura suave. C: Corte transversal de tallo. D: Corte transversal de hoja. E: Corte transversal de sépalo. F: Corte transversal de pétalo. Abreviaturas: col, colpas; end, endospermo; ent, endotesta; ext, exotesta; per, peridermis; cam, cambiun; xi, xilema; flo, floema; fi, fibra; pm, parénquima medular; hv, haz vascular; pg, pelo glandular; pe, parénquima esponjoso; ep, epidermis; cr, cromoplastos; colen, colénquima. 


\section{AGRADECIMIENTOS}

Agradecemos a la licenciada Maritza Martínez por sus comentarios en el texto y al licenciado Leonel Zúniga por su apoyo en la toma de fotografías.

\section{BIBLIOGRAFÍA}

Chizmar, Carla; Lu, Allys; Correa A., Mireya D. (2009). Plantas de uso folclórico y tradicional en Panamá. Costa Rica: Instituto Nacional de Biodiversidad.

Gentry, A. H. \& Tomb, S. (1979). Taxonomic Implications of Bignoniaceae Palynology. Annals of the Missouri Botanical Garden, (66)4, 756-777.

González, A.M. (2013). Indumento, nectarios extraflorales y anatomía foliar en Bignoniáceas de la Argentina. Boletín de la Sociedad Argentina de Botánica, 48(2), 221-245.

Hernández, E. y Campos, A. (2007). Utilización del zumo de jícaro (Crescentia cujete $L$ ) en el tratamiento de la dermatomicosis en terneros de la raza reina en la finca Santa Rosa de la UNA. Tesis. Universidad Nacional Agraria, Nicaragua. House, P. R.; Lagos-Witte, S.; Ochoa, L.; Torres, C.; Mejía, T. y Rivas, M. (1995). Plantas medicinales comunes de Honduras. Tegucigalpa.

Jarquín, J. (2012). Propuesta de inversión en sistemas silvopastoriles sostenibles en bosques de trópico seco. Nicaragua. Red Académica Iberoamericana Local Global, 5(15), 13.

Jiménez, O. (2008). Índice de confort de la vegetación. Revista Nodo, 3(5), 49-70.

Meyrat, A. (1983). El jícaro sabanero (Crescentia alata HBK), un potencial económico a desarrollar en las zonas secas de Nicaragua. Nicaragua: UNAN.

Mitra, K. (1968). Pollen morphology in Bignoniaceae in relation to taxonomy. Bulletin of the Botanical Survey of India, 10, 319-326.

Rivera, G.L. (2000). Nuptial nectary structure of Bignoniaceae from Argentina. Darwiniana, 38(3-4), 227-239.

Roth, I. (1969). Estructura anatómica de la corteza de algunas especies arbóreas de Bignoniaceae. Acta Botánica Venezuélica, (4)1/4, 157-174.

Seibert, R. J. (1948). The use of glands in a taxonomic consideration of the family Bignoniaceae. Annals Missouri Botanical Garden, 35, 123-137.

Simaj, C. y García, J. R. (2004). Utilización del zumo de guirra (Crescentia cujete) en el tratamiento de endometritis en hembras bovinas. Revista Electrónica de Veterinaria, (IX)7, 1-11.

Solares F. A. (2004). Etnobotánica y usos potenciales del cirián (Crescentia alata, H.B.K.) en el estado de Morelos. Polibotánica, 18, 13-31.

Urban, I. (1916). Uber Ranken und Pollen der Bignoniaceae. Berichte der Deutschen Botanischen Gesellschaft, 34, 723-758.

Zapater, M. A.; Califano, L. M.; Del Castillo, E. M.; Quiroga, M. A. y Lozano, E. A. (2009). Las especies nativas y exóticas de Tabebuia y Handroanthus (Tecomeae, Bignoniaceae) en Argentina. Darwiniana, 47, 185-22. 